\title{
Rare Concurrence of Congenital Muscular Torticollis and a Malignant Tumor in the Same Sternocleidomastoid Muscle
}

\author{
Yul-Hyun Park, $\mathrm{MD}^{1}$, Chul-Ho Kim, $\mathrm{MD}, \mathrm{PhD}^{2}$, Jang-Hee Kim, MD, PhD ${ }^{3}$, \\ Jun-Eun Park, $\mathrm{MD}, \mathrm{PhD}^{4}$, Shin-Young Yim, $\mathrm{MD}, \mathrm{PhD}^{1}$
}

\author{
${ }^{1}$ The Center for Torticollis, Department of Physical Medicine and Rehabilitation, \\ Departments of ${ }^{2}$ Otolaryngology, ${ }^{3}$ Pathology, and ${ }^{4}$ Pediatrics, Ajou University School of Medicine, Suwon, Korea
}

\begin{abstract}
While congenital muscular torticollis (CMT) can occur along with other conditions, such as clavicle fracture or brachial plexus injury, these conditions exist outside the sternocleidomastoid muscle (SCM). We present a rare case with concurrence of CMT and a malignant tumor inside the same SCM, along with serial clinical and radiological findings of the atypical features of CMT. The malignant tumor was in fact a low-grade fibromyxoid sarcoma. To the best of our knowledge, the current case is the first of a concurrent condition of CMT inside the SCM. This case suggests that concurrent conditions could exist either inside or outside the SCM with CMT. Therefore, a thorough evaluation of SCM is required when subjects with CMT display atypical features, such as the increase of mass or poor response to conservative therapy. In that case, appropriate imaging modalities, such as ultrasonogram or magnetic resonance imaging, are useful for differential diagnosis.
\end{abstract}

Keywords Torticollis, Congenital torticollis, Fibrosarcoma, Magnetic resonance imaging

\section{INTRODUCTION}

Congenital muscular torticollis (CMT) is one of the most common musculoskeletal problems in children. The incidence of CMT is as high as $3.92 \%$ in neonates. CMT is characterized by a thickening and/or tightness of the unilateral sternocleidomastoid muscle (SCM), which limits neck motion [1]. CMT can occur along with various conditions, such as brachial plexus injury or unilateral developmental dysplasia of the hip and these concurrent conditions exist outside the SCM $[2,3]$.

We experienced a rare case of concurrence of CMT and low-grade fibromyxoid sarcoma (LGFMS) in the same SCM. LGFMS is a malignant tumor that usually occurs in the deep soft tissue of the extremities and trunk. LGFMS needs radical excision due to a potential for recurrence

Received February 27, 2017; Accepted May 26, 2017

Corresponding author: Shin-Young Yim

The Center for Torticollis, Department of Physical Medicine and Rehabilitation, Ajou University School of Medicine, 164 Worldcup-ro, Yeongtong-gu, Suwon 16499, Korea. Tel: +82-31-219-5284, Fax: +82-31-219-5209, E-mail: syyim@ajou.ac.kr

ORCID: Yul-Hyun Park (http://orcid.org/0000-0003-3103-293X); Chul-Ho Kim (http://orcid.org/0000-0002-2161-4488); Jang-Hee Kim (http://orcid. org/0000-0001-5825-1361); Jun-Eun Park (http://orcid.org/0000-0003-4292-3500); Shin-Young Yim (http://orcid.org/0000-0002-8521-6331).

(c) This is an open-access article distributed under the terms of the Creative Commons Attribution Non-Commercial License (http://creativecommons.org/ licenses/by-nc/4.0) which permits unrestricted noncommercial use, distribution, and reproduction in any medium, provided the original work is properly cited. Copyright $\odot 2018$ by Korean Academy of Rehabilitation Medicine 
and metastasis [4]. To the best of our knowledge, there has been no report on concurrence of CMT and malignant tumors including LGFMS in the same SCM. Here we report this case along with clinical implications.

\section{CASE REPORT}

A 4-month-old girl visited our clinic with the diagnosis of right CMT and right brachial plexus injury. The ultrasonogram done at the previous hospital showed diffuse thickening and heterogenous hyperechogenicity in the right SCM compared with the left SCM, suggesting right CMT (Fig. 1A, 1B). She was born through a vaginal delivery at full term with a birth weight of $3,640 \mathrm{~g}$. The delivery was complicated with cephalhematoma and right bra- chial plexus injury. On the first visit, clinical examination revealed tight and thick right SCM. She received physical therapy for 3 months in the previous hospital, but there was no improvement in the range of neck motion. We measured her neck rotation with an arthrodial protractor. For that, she lay supine with the shoulders stabilized and the examiner supported the head and neck in the neutral position. Lateral flexion was measured with a large protractor. There were $50^{\circ}$ of neck rotation toward right side and $40^{\circ}$ of neck lateral flexion toward left side, ending up with right torticollis. She also showed weakness of right shoulder girdle muscles and elbow flexors, showing grade 2 on pediatric functional muscle testing. By inducing the patient's developmental reflexes, we observed the reaction and evaluated to grade 2 that only partial move-
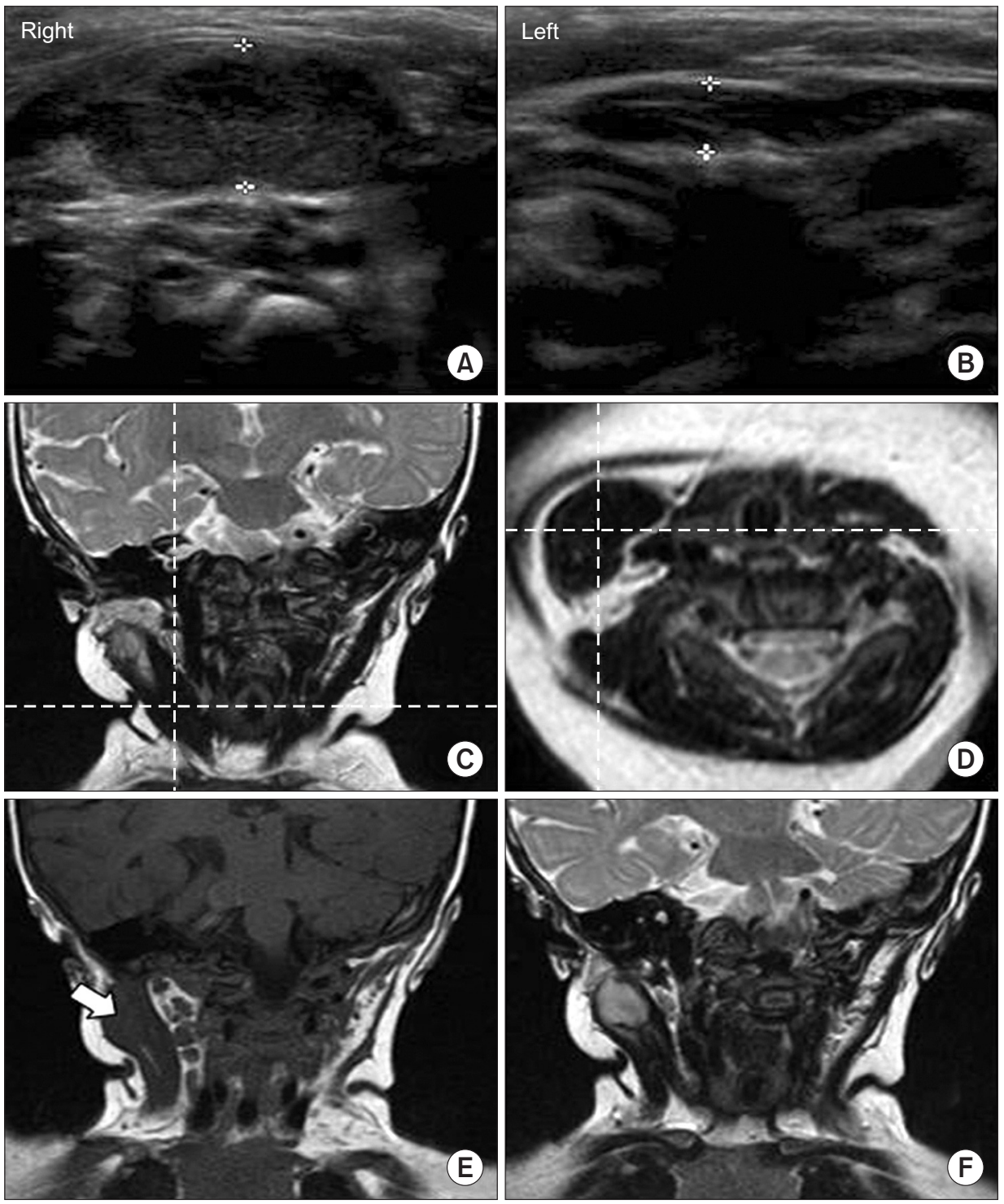

Fig. 1. Radiologic findings of the sternocleidomastoid muscle (SCM) with of congenital muscular torticollis (CMT). (A, B) Ultrasonogram shows diffuse thickening and heterogenous hyperechogenicity in the right SCM when compared with the left SCM, with $1.03 \mathrm{~cm}$ and $0.46 \mathrm{~cm}$ of the thickness for the right and left SCM, respectively. (C) Magnetic resonance imaging (MRI) of the neck shows diffuse thickening of the right SCM with low signal intensity within the right SCM on coronal T2-weighted image. (D) Axial T1-weighted image also shows asymmetric diffuse thickening of the right SCM with low signal intensity at the same level of the coronal view, suggestive of CMT. (E) Coronal T1-weighted image shows a mass with diameter of 1.5 $\mathrm{cm}$ with low signal intensity in the superior portion of the right SCM (white arrow). (F) Coronal T2weighted image shows high signal intensity at the same mass. 
ment was possible [5]. She showed shortening of the left leg in comparison to the right, and was diagnosed with developmental dysplasia of the left hip by an ultrasonogram. The alpha angle of the left hip was $45^{\circ}$ and the beta angle was $62^{\circ}$. She was treated with a Pavlik harness for 4 months. With the diagnosis of right CMT and brachial plexus injury, she received physical therapy for CMT and brachial plexus injury. After 3 months of treatment, the strength of shoulder girdle muscles and elbow flexors was improved from grade 2 to grade 4 , which can tolerate resistance without breaking the test position on the pediatric functional muscle testing [5]. However, she did not show any significant improvement in the range of neck motion. The patient showed too much resistance to continue the stretching exercise, so she did not respond to physical therapy for 7 months. Therefore, the neck magnetic resonance imaging (MRI) was performed at the age of 8 months in order to determine whether surgical release was required. The neck MRI showed diffuse thickening of the right SCM with low signal intensity within right SCM on both T1- and T2-weighted images, suggestive of right CMT (Fig. 1C, 1D). In addition, a mass with diameter of $1.5 \mathrm{~cm}$ with low signal intensity on T1weighted image and high-signal intensity on T2-weighted image was found in the superior portion of the right SCM, unlike typical findings of CMT (Fig. 1E, 1F).

At the age of 9 months, unipolar release of both the sternal and clavicular head of the right SCM was performed just above right clavicle for management of CMT to prevent musculoskeletal complications secondary to CMT. A needle biopsy was also performed on the mass in the superior portion of the right SCM. Microscopic examination of a hematoxylin and eosin stained biopsy specimen revealed diffuse spindle cell proliferation with fibrosis, consistent with CMT. As the pathologic findings appeared to be compatible with CMT, she received postoperational physical therapy without considering surgical treatment for the mass lesion in the superior portion and was followed up in the outpatient clinic.

Four months after surgery, she had gained full range of neck motion, but the mass in the superior portion of the right SCM had been growing. Neck MRI was performed to confirm the possibility of malignant neoplasm in the rapidly growing mass. Because the neck MRI of CMT patients can demonstrate high signal intensity on T2weighted images caused by edematous condition for 1 year, we additionally conducted gadolinium-enhanced imaging. The mass in the superior portion of the right SCM on the follow-up MRI showed iso- to low- signal intensity on T1-weighted image and high signal intensity on coronal T2-weighted image and gadoliniumenhanced T1-weighted image, along with increased size of the mass from $2.1 \times 2.8 \times 2.5 \mathrm{~cm}^{3}$ to $2.8 \times 4.1 \times 4.2 \mathrm{~cm}^{3}$ (Fig. $2 \mathrm{~A}, 2 \mathrm{~B}, 2 \mathrm{C})$. A multidisciplinary team including a pediatric oncologist, otolaryngologist, and radiation oncologist concluded that the possibility of low-grade malignant tumor indicating rapid growing could not be excluded, and so radical excision of the mass was performed.

The surgical specimen revealed a circumscribed yellow to gray solid mass with focal myxoid change (Fig. 2D). Microscopically, the tumor showed myxoid and fibrous areas composed of spindle cells (Fig. 2E, 2F). In areas of hypercellularity, it showed positive immunohistochemical staining for Ki-67, which is a marker of proliferating cells (Fig. 2G). Tumor cells were negative for CD34, which is one of the markers of solitary fibrous tumor. Tumor invasion to surrounding soft tissue was present but there was no involvement of resection margin. On the basis of these findings, the excised mass was proven to be LGFMS. G-banding karyotyping using the primary-cultured cells from the surgical specimen showed $47, \mathrm{XX}, \mathrm{t}(4 ; 18)$ (q27;q23),+14[3]/46,XX[27] (Fig. 2H, 2I). Positron emission tomography-computed tomography performed at the age of 15 months revealed no evidence of metastasis. On monthly follow-ups without adjuvant radiotherapy over 19 months, there was no evidence of recurrence on MRI.

\section{DISCUSSION}

About $90 \%$ or more of CMT cases are cured with only stretching exercises, but about $10 \%$ of CMT cases do not respond to this method. The cases of CMT showing an unfavorable response to physical therapy have a large amount of dense connective tissue within the SCM. In these cases, neck MRI is useful for evaluating fibrosis and determining whether surgical release is needed [6]. In the current case, neck MRI showed diffuse dense connective tissue in the lower portion of the SCM and operation was performed. There was also a mass with high signal intensity on T2-weighted image in the superior portion of the same SCM. The neck MRI of CMT patients 

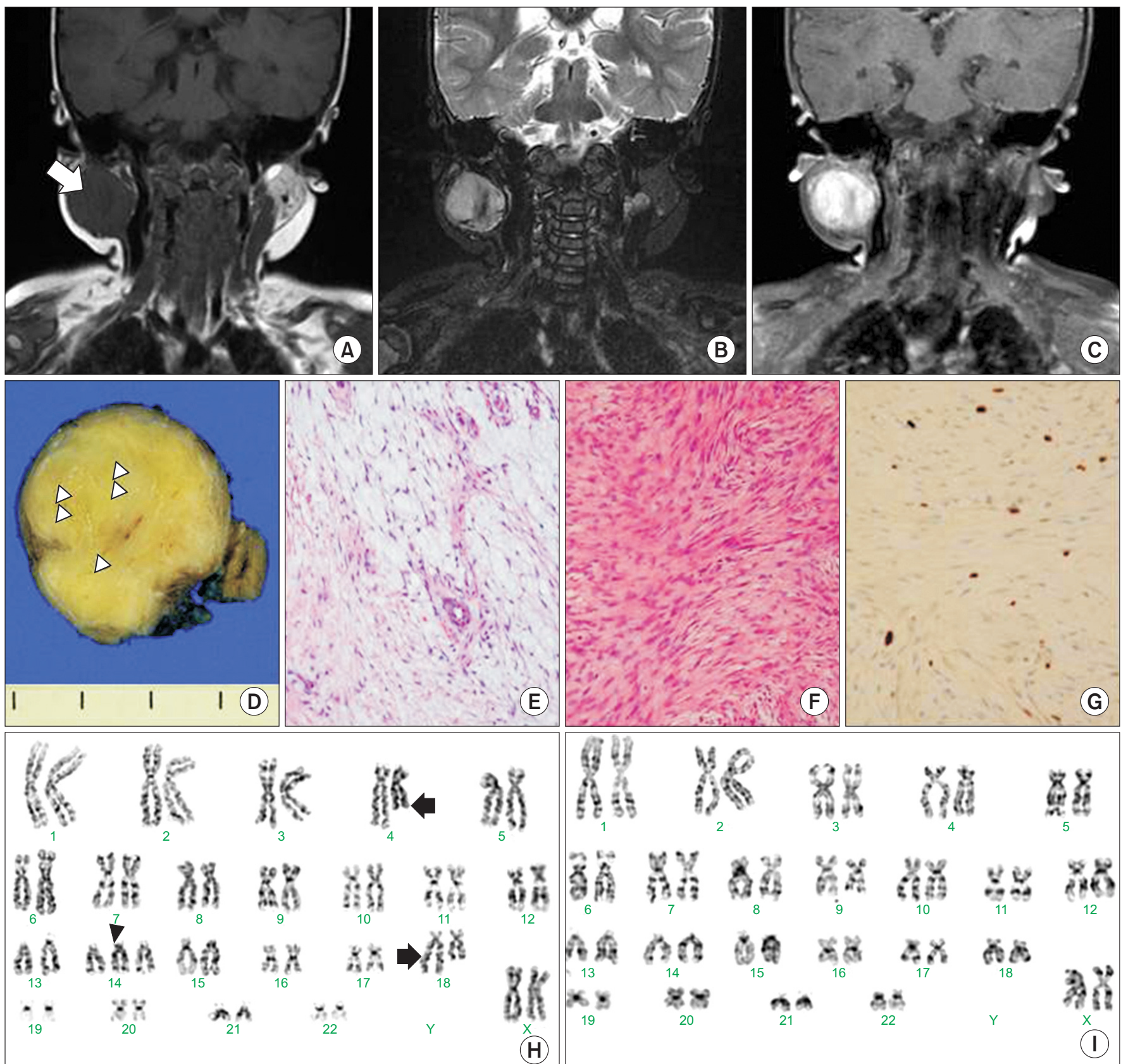

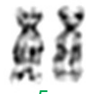

Fig. 2. Radiologic and histopathologic findings of low-grade fibromyxoid sarcoma (LGFMS). (A-C) The mass on the followup magnetic resonance imaging (MRI) showed iso- to low-signal intensity on T1-weighted image and high signal intensity on coronal T2-weighted image and the gadolinium-enhanced T1-weighted image along with increased size of the mass. (D) The cross-section of the mass shows circumscribed light yellow to gray solid mass with focal myxoid change (white arrow heads). (E, F) The histopathologic findings of the mass showed myxoid and fibrous areas which were mainly composed of spindle cells (H\&E, $\times 200)$. (G) In areas of hypercellularity, it shows positive immunohistochemical staining for Ki-67 which is one of markers of proliferating cells (anti-Ki-67, $\times 200$ ). (H, I) G-banding karyotyping of primary-cultured cells shows somatic mosaicism with the 3 cells with $47, \mathrm{XX}, \mathrm{t}(4 ; 18)(\mathrm{q} 27 ; \mathrm{q} 23),+14$ and 27 cells with $46, \mathrm{XX}$ among 30 cells.

in the early stage can demonstrate high signal intensity on T2-weighted image due to edematous condition and the needle biopsy specimen for this mass was compatible with CMT [7]. Therefore, we decided to observe the mass. However, there were limitations in making an accurate diagnosis because the specimen was taken with needle 
biopsy and the mass was rapidly growing. The follow-up neck MRI still showed high signal intensity on T2-weighted image and gadolinium-enhanced T1-weighted image. We could not rule out malignancy and performed radical excision of the mass. The excised mass turned out to be a malignant tumor.

While CMT is known to concur with some conditions, such as clavicular fracture, brachial plexus injury or unilateral developmental dysplasia of the hip, these concurrent conditions exist outside the SCM. The current case showed concurrence of CMT and a malignant tumor in the same SCM. Even though this condition seems to be rare, the current case carries a couple of clinical implications. First, concurrent conditions of CMT could exist in the same SCM with CMT. Second, an increase in size of a mass in the SCM after neonatal period suggests that the mass might not be CMT. The SCM with CMT could increase in its thickness, volume, or both during the early neonatal period, probably due to ongoing edema or inflammation of the involved SCM. However, the mass in the SCM stabilizes in size for few months and gradually diminishes by 4-8 months of age, thus it is less likely for CMT to increase the size of mass after neonatal period [8]. Third, high signal intensity on T2-weighted MRI image of the SCM is not a typical finding of CMT in chronic stage. The current case showed two different findings of the same SCM in the neck MRI. There was low signal intensity on both T1- and T2-weighted images in the lower twothirds of the SCM, which was compatible with CMT. In addition, there was a mass with high signal intensity on a T2-weighted image in the upper one-third of the same SCM and it turned out to be LGFMS. Neck MRI of CMT in chronic stage do not show high signal intensity on both T1- and T2-weighted images, since the major histopathologic findings of CMT is interstitial fibrosis, which leads to a reduction of the density of hydrogen ions [6].

In the current case, the patient was finally diagnosed with CMT concurrent with LGFMS in the same SCM. LGFMS is a soft tissue tumor [9]. On microscopic examination, LGFMS has fibrous and myxoid components along with spindle cells. This heterogeneous histological appearance makes the diagnosis challenging. The somatic cells of LGFMS have been reported to be associated with recurrent chromosomal translocation $\mathrm{t}(7 ; 16)(\mathrm{q} 33 ; \mathrm{p} 11)$ or $\mathrm{t}(11 ; 16)(\mathrm{p} 11 ; \mathrm{p} 11)$. These chromosomal translocations induce fusion genes such as FUS-CREB3L2 or FUS-
CREB3L. However, a lack of those chromosomal rearrangements does not exclude the diagnosis of LGFMS, since cases of LGFMS without those chromosomal rearrangements have been reported [4]. In the current case, the primary-cultured cells of the excised mass of LGFMS showed low-grade somatic mosaicism with $47, \mathrm{XX}, \mathrm{t}(4 ; 18)$ (q27;q23),+14[3]/46,XX[27]. We could not find any report regarding clinical implications of these rearrangements in the aspect of pathogenesis with LGFMS.

One case report describing desmoid fibromatosis in the SCM, which was initially misdiagnosed as CMT [10]. However, there has been no report on the concurrent conditions of CMT with SCM. The current case seems to be the first report on the concurrence of CMT and LGFMS in the same SCM. Therefore, a thorough evaluation of SCM is required for subjects with CMT, who show atypical features or a poor response to conservative therapy. A thorough assessment using appropriate imaging modalities, such as ultrasonogram or MRI, is important for differential diagnosis.

\section{CONFLICT OF INTEREST}

No potential conflict of interest relevant to this article was reported.

\section{REFERENCES}

1. Chen MM, Chang HC, Hsieh CF, Yen MF, Chen TH. Predictive model for congenital muscular torticollis: analysis of 1021 infants with sonography. Arch Phys Med Rehabil 2005;86:2199-203.

2. Walsh JJ, Morrissy RT. Torticollis and hip dislocation. J Pediatr Orthop 1998;18:219-21.

3. Hervey-Jumper SL, Justice D, Vanaman MM, Nelson VS, Yang LJ. Torticollis associated with neonatal brachial plexus palsy. Pediatr Neurol 2011;45:305-10.

4. Mastoraki A, Strigkos T, Tatakis FP, Christophi A, Smyrniotis V. Recurrent low-grade fibromyxoid sarcoma of the neck: report of a case and review of the literature. Indian J Surg Oncol 2015;6:296-9.

5. Seo HJ, Kim JH. The reliability of the pediatric functional muscle testing in children with developmental delay. J Korea Phys Ther 2015:27:183-9.

6. Hwang JH, Lee HB, Kim JH, Park MC, Kwack KS, Han $\mathrm{JD}$, et al. Magnetic resonance imaging as a determi- 
nant for surgical release of congenital muscular torticollis: correlation with the histopathologic findings. Ann Rehabil Med 2012;36:320-7.

7. Entel RJ, Carolan FJ. Congenital muscular torticollis: magnetic resonance imaging and ultrasound diagnosis. J Neuroimaging 1997;7:128-30.

8. Jaber MR, Goldsmith AJ. Sternocleidomastoid tumor of infancy: two cases of an interesting entity. Int J Pe- diatr Otorhinolaryngol 1999;47:269-74.

9. Evans HL. Low-grade fibromyxoid sarcoma: a report of two metastasizing neoplasms having a deceptively benign appearance. Am J Clin Pathol 1987;88:615-9.

10. Ekinci S, Karnak I, Tanyel FC. Infantile fibromatosis of the sternocleidomastoid muscle mimicking muscular torticollis. J Pediatr Surg 2004;39:1424-5. 